\title{
ON THE IMAGE SIZE OF SINGULAR MAPS. I
}

\author{
S. M. BATES
}

(Communicated by Andrew M. Bruckner)

\begin{abstract}
For fixed integers $n>m>r_{l}+1>0$, we establish a sharp upper bound for the Hölder differentiability class of singular maps $f: \mathbf{R}^{n} \rightarrow \mathbf{R}^{m}$ of subrank $r_{l}$ whose images have positive Lebesgue $m$-measure.
\end{abstract}

\section{INTRODUCTION}

An important result in analysis, known as the Morse-Sard Theorem, states that the set of critical values of a function $f \in C^{k}\left(\mathbf{R}^{n}, \mathbf{R}^{m}\right)$ has measure zero if $k \geq \max (n-m+1,1)$. The necessity of the above differentiability requirement was first established by [Whitney, 1935], who produced a technique for constructing a function $f \in C^{1}\left(\mathbf{R}^{n}, \mathbf{R}\right)$ which is not constant on a connected set of critical points. Using this result, one can easily construct a map $g \in C^{1}\left(\mathbf{R}^{n+q}, \mathbf{R}^{q}\right)$ whose set of critical values contains an open set by defining $g\left(x_{1}, \ldots, x_{n+q}\right)=\left(f\left(x_{1}, \ldots, x_{n+1}\right), x_{n+2}, \ldots, x_{n+q}\right)$. Nevertheless, such examples always have regular points arbitrarily close to their set of critical points; this fact suggests the following question, which first appeared in [Hirsch, 1976]:

Can the image of a singular $C^{k}$ map $f: \mathbf{R}^{n} \rightarrow \mathbf{R}^{m}$ contain an open set?

If $k=1$, and either $n \leq m$ or $m=1$, the answer is evidently "no," whereas [Kaufman, 1979] has given examples of rank 1 maps whose images contain open sets for $k=1, n=m+1$.

In the following, we modify recent Hölder calculus results of [Norton, 1986] to show that, for integers $n>m>r_{l}+1>0$, the number $s_{0}=\left(n-r_{l}\right) /\left(m-r_{l}\right)$ has the following properties:

(i) If $s=s_{0}$, and $f: \mathbf{R}^{n} \rightarrow \mathbf{R}^{m}$ is a singular $C^{s+}$ map with subrank $r_{l}$ (see definitions below), then $f\left(\mathbf{R}^{n}\right)$ has Lebesgue $m$-measure zero.

(ii) If $s<s_{0}$, there exists a singular $C^{s}$ map $f: \mathbf{R}^{n} \rightarrow \mathbf{R}^{m}$ with subrank $r_{l}$ whose image contains an open set.

For the special case of singular mappings, the bound $s_{0}$ improves estimates for general maps published in [Norton, 1986] and [Federer, 1969].

Received by the editors August 23, 1990.

1980 Mathematics Subject Classification (1985 Revision). Primary 26B35; 57R70, 58C25, $58 \mathrm{C} 27$.

The author holds an NSF graduate fellowship. 
Notations and definitions. In the following, $l_{m}$ and $\mathscr{H}^{t}$ denote the Lebesgue measure on $\mathbf{R}^{m}$ and the $t$-dimensional Hausdorff (outer) measure, respectively, while $E$ always denotes an open subset of some specified Euclidean space. A subset $A \subset E$ is $t$-finite if $\mathscr{H}^{t} A<\infty$ and $t$-sigmafinite if $A$ is a countable union of $t$-finite sets.

If $m \leq n$ and $f: E \subset \mathbf{R}^{n} \rightarrow \mathbf{R}^{m}$ is a $C^{1}$ map, then we decompose $E$ as $E=\bigcup_{r=0}^{m} E_{r}$, where $E_{r}=\{x \in E: \operatorname{rank} D f(x)=r\}$, and define $r_{\max }=\max \{\operatorname{rank} D f(x): x \in E\}$ to be the rank of $f$ (on $E$ ). $f$ is singular if $r_{\max }<m$; if rank $D f$ assumes more than one value on $E$, the subrank of $f$ is $r_{l}=\max \left\{\operatorname{rank} D f(x): x \in E \backslash E_{r_{\max }}\right\}$. Any subset $A \subset E$ satisfying $\max \{\operatorname{rank} D f(x): x \in A\} \leq r$ is called a set of rank $r$ for $f$.

For real $s \geq 1$, let $[s]$ denote the greatest integer $\leq s$. The map $f$ above is said to be $C^{s}$ if $f \in C^{[s]}$ and for every compact neighborhood $V \subset E$, there exists a number $M>0$ such that $\left\|D^{[s]} f(x)-D^{[s]} f(y)\right\| \leq M|x-y|^{s-[s]}$ for all $x, y \in V . f$ is $C^{s+}$ if $f \in C^{[s]}$, and for every compact neighborhood $V \subset E$, there exists a continuous monotone function $\varepsilon: \mathbf{R} \rightarrow \mathbf{R}$ with $\varepsilon(0)=0$ and $\left\|D^{[s]} f(x)-D^{[s]} f(y)\right\| \leq \varepsilon(|x-y|)|x-y|^{s-[s]}$ for all $x, y \in V$. (Note: $\left.\bigcup_{s^{\prime}>s} C^{s^{\prime}} \subset C^{s+}\right)$. text.

Finally, $\left\{M_{i}\right\}$ always denote positive real constants chosen according to con-

\section{THE HÖLDER BOUND}

Note that a routine application of the Morse-Sard Theorem shows that $l_{m} f\left(\mathbf{R}^{n}\right)=0$ for any singular map $f \in C^{1}\left(\mathbf{R}^{n}, \mathbf{R}^{m}\right)$ when $n \leq m$, while the Rank Theorem implies the same conclusion for a singular map $f$ between arbitrary Euclidean spaces such that $\operatorname{rank} D f$ is constant. Thus we assume throughout that $n>m>1$ and consider only singular maps for which $r_{l}$ exists. We shall rely heavily on the following fact due to [Norton, 1986]:

Lemma 1 (Norton). Suppose $f \in C^{s+}\left(\mathbf{R}^{n}, \mathbf{R}^{m}\right)$ and $A$ is $a(s(m-r)+r)-$ sigmafinite set of rank $r$ for $f$, with $r<m$. Then $l_{m} f(A)=0$.

We now derive our main result:

Theorem 1. Suppose $n, m$, and $r_{l}$ are integers satisfying $n>m>r_{l}+1>0$, and define $s_{0}=\left(n-r_{l}\right) /\left(m-r_{l}\right)$. Let $E \subset \mathbf{R}^{n}$ be open.

(i) If $f: E \rightarrow \mathbf{R}^{m}$ is a singular $C^{s+}$ map with subrank $r_{l}$, then $s=s_{0}$ implies $l_{m} f(E)=0$.

(ii) For each $s<s_{0}$, there exists a singular $C^{s}$ map $f: \mathbf{R}^{n} \rightarrow \mathbf{R}^{m}$ with subrank $r_{l}$ whose image contains an open set.

Proof. Let $E=\bigcup_{r=0}^{m} E_{r}$ as described above, $s=s_{0}$. We show that $l_{m} f\left(E_{r}\right)=0$ for $r=0, \ldots, m$.

1. Since any subset of $\mathbf{R}^{n}$ is $n$-sigmafinite and $s=\left(n-r_{l}\right) /\left(m-r_{l}\right) \geq$ $(n-r) /(m-r)$, it follows from Lemma 1 that $l_{m} f\left(E_{r}\right)=0$ for $r \in\left\{0, \ldots, r_{l}\right\}$.

2. For $r=r_{\max }$, we note that since $r$ is maximal, $E_{r}$ is evidently open in $E$. Since $\operatorname{rank} D f=r<m$ on $E_{r}$, the Rank Theorem asserts that $E_{r}$ is the union of countably many open subsets $\left\{A_{i}\right\}$ such that each $f\left(A_{i}\right)$ is a $C^{1}$ $r$-dimensional submanifold of $\mathbf{R}^{m}$. Thus $l_{m} f\left(A_{i}\right)=0$ for all $i$, from which it follows that $l_{m} f\left(E_{r}\right)=l_{m} \cup f\left(A_{i}\right)=0$. 
3. Since by definition $E_{r}=\varnothing$ for $r_{l}<r<r_{\max }$ and $r_{\max }<r \leq m$, this proves part (i).

The proof of statement (ii) is the topic of §III below.

By definiton, $r_{l} \leq m-2$. Thus we obtain:

Corollary 1. The image of any $C^{s+}$ singular map $f: E \subset \mathbf{R}^{n} \rightarrow \mathbf{R}^{m}$ has $m$ measure zero whenever $s \geq 1+(n-m) / 2$.

If the Hölder differentiability class of a singular map with nonnull image is known, Theorem 1 yields the following necessary rank condition:

Corollary 2. Suppose $f: E \subset \mathbf{R}^{n} \rightarrow \mathbf{R}^{m}$ is a $C^{s+}$ singular map such that $l_{m} f(E)>0$. Then the subrank $r_{l}$ of $f$ exists and satisfies

$$
(s m-n) /(s-1)<r_{l} \leq m-2 .
$$

Furthermore, if $n>m>1$, then for any nonnegative integer $r \in$ $((s m-n) /(s-1), m-2]$ there exists a singular map $g: \mathbf{R}^{n} \rightarrow \mathbf{R}^{m}$ for which $r_{l}=r$, and Int $g\left(\mathbf{R}^{n}\right) \neq \varnothing$.

\section{SHARPNESS OF BOUND}

To prove statement (ii) of Theorem 1, we construct, for integers $n>m>$ 1 , and for real $s \in(1, n / m)$, a $C^{s}$ function $f: E \subset \mathbf{R}^{n} \rightarrow \mathbf{R}^{m}$ such that Int $f(E) \neq \varnothing$, although $\operatorname{rank} D f(x) \leq 1$ for all $x \in E$. Intuitively, this is the most geometrically bizarre class of such phenomena, from which examples of less complex behavior may be expected to follow. Indeed, assuming the construction for the time being, choose integers $n, m$, and $r$ with $0<r+1<m<n$, and a real $s \in(1,(n-r) /(m-r))$. Let $f: E \subset \mathbf{R}^{n-r} \rightarrow$ $\mathbf{R}^{m-r}$ satisfy $f \in C^{s}, \operatorname{rank} D f(x) \leq 1$ for all $x \in E$, and Int $f(E) \neq \varnothing$. Then the singular $C^{s}$ map $g: E \times \mathbf{R}^{r} \rightarrow \mathbf{R}^{m}$ defined by $\left(x_{1}, \ldots, x_{n}\right) \mapsto$ $\left(f\left(x_{1}, \ldots, x_{n-r}\right), x_{m+1-r}, \ldots, x_{m}\right)$ has subrank $r$ on $E \times \mathbf{R}^{r}$, while clearly Int $g\left(E \times \mathbf{R}^{r}\right)=(\operatorname{Int} f(E)) \times \mathbf{R}^{r} \neq \varnothing$.

Construction of $f$ : We modify a technique of [Kaufman, 1979] whose notation we retain for continuity. Let $n>m>1$ be fixed integers, and let $P \geq 1$ denote the greatest integer less than $n / m$. Fix $s \in(P, n / m)$.

1. A Cantor set in $\mathbf{R}^{n}$ : For $\beta=2^{-n / s m}$, we define the following method for constructing $2^{n}$ cubes within any cube in $\mathbf{R}^{n}$ :

Supposing $I \subset \mathbf{R}^{n}$ is a cube of side $L>0$ defined by the inequalities $\left|x_{i}-c_{i}\right| \leq L / 2$, we specify $2^{n}$ subcubes within $I$ via the inequalities $\left|x_{i}-c_{i} \pm L / 4\right| \leq \beta L / 2$. Note that each subcube is separated from all other subcubes and the boundary of $I$ by a distance $\geq L(1 / 4-\beta / 2)$.

Let $I_{0} \subset \mathbf{R}^{n}$ denote the cube defined by $\left|x_{i}\right| \leq 1$. By repeated application of the above process, we obtain subcubes $\left\{I\left(a_{1}, \ldots, a_{k}\right)\right\}$, where $\left\{I\left(a_{1}\right)\right\}$ are the $2^{n}$ subcubes constructed within $I_{0}$, etc., and $1 \leq a_{i} \leq 2^{n}$ for all $i \in$ $\{1, \ldots, k\}$. Furthermore, we require that the $2^{n}$ subcubes $\left\{I\left(a_{1}, \ldots, a_{k+1}\right)\right\}$ constructed within the cube $I\left(a_{1}, \ldots, a_{k}\right)$ be labelled in the unique way which corresponds homothetically to a chosen labelling of the $\left\{I\left(a_{1}\right)\right\}$, that is, the cube $I\left(a_{1}, \ldots, a_{k}\right)$ is a scaled-down copy of $I_{0}$ such that the subcube $I\left(a_{1}, \ldots, a_{k}, a\right)$ corresponds to the subcube $I(a)$ for $1 \leq a \leq 2^{n}$. For integers $k \leq l$, the boundaries of distinct cubes $I\left(a_{1}, \ldots, a_{k}\right)$ and $I\left(a_{1}^{\prime}, \ldots, a_{l}^{\prime}\right)$ are separated by a distance $\geq \beta^{k}(1 / 4-\beta / 2)$. 
We now let $C(\beta)$ denote the Cantor set defined by the cubes, i.e. the set of points in $I_{0}$ contained in infinitely many subcubes belonging to the above construction.

2. Mapping of $C(\beta)$ : Let $R_{0} \subset \mathbf{R}^{m}$ denote the cube defined by $\left|x_{i}\right| \leq 1$, $1 \leq i \leq m$, and let $\left\{e_{i}\right\}_{i=1}^{m}$ be the standard basis of $\mathbf{R}^{m}$. Define $\left\{R\left(a_{1}\right)\right\}, 1 \leq$ $a_{1} \leq 2^{n}$, to be the $2^{n}$ congruent $m$-rectangles constructed by evenly partitioning $R_{0}$ with $(m-1)$-planes of unit normal $e_{1}$. Similarly, for $k \in \mathbf{Z}^{+}$, we construct $\left\{R\left(a_{1}, \ldots, a_{k+1}\right)\right\}$ within $R\left(a_{1}, \ldots, a_{k}\right)$ by evenly partitioning the the latter set with $(m-1)$-planes having unit normal $e_{((k-1) \bmod m)+1}$ and subject our labelling to the requirement that the $e_{((k-1) \bmod m)+1}$-coordinate of the midpoint of $R\left(a_{1}, \ldots, a_{k+1}\right)$ increase as the index $a_{k+1}$ increases from 1 to $2^{n}$. Note that each $R\left(a_{1}, \ldots, a_{k}\right)$ has diameter $\leq M_{1} 2^{-(n / m) k}$.

We now let $h: C(\beta) \rightarrow R_{0}$ be the (surjective) map defined by the requirement that $h\left(C(\beta) \cap I\left(a_{1}, \ldots, a_{k}\right)\right) \subset R\left(a_{1}, \ldots, a_{k}\right)$ for all $1 \leq a_{i} \leq 2^{n}, \quad 1 \leq$ $i \leq k$, and $k \in \mathbf{Z}^{+}$.

3. Extension of $h$ : The extension of $h$ to a function $f: I_{0} \rightarrow R_{0}$ is carried out by first defining $f$ on the closure of the set $I_{0} \backslash \bigcup I\left(a_{1}\right)$. A scaling relation then enables us to specify $f$ on the remainder of $I_{0} \backslash C(\beta)$.

(i) We require that $f$ be constant on a neighborhood of each boundary $\partial I\left(a_{1}\right)$ and that its value equal the midpoint of the set $R\left(a_{1}\right)$. More precisely, define $\varphi:\left\{1, \ldots, 2^{n}\right\} \rightarrow \mathbf{R}$ by $\varphi(i)=2 i-\left(2^{n}+1\right)$ and let $S$ denote the closure of the set $I_{0} \backslash \bigcup I\left(a_{1}\right)$. Choose a $C^{\infty}$ function $\lambda: S \rightarrow[-1 / 2,1 / 2]$ such that for $1 \leq a_{1} \leq 2^{n}$,

(a) $\lambda(x)=2^{-(n+1)} \varphi\left(a_{1}\right)$ if $d\left(x, \partial I\left(a_{1}\right)\right)<\beta(1 / 4-\beta / 2) / 10$.

(b) $\lambda(x)=0$ if $d\left(x, \bigcup \partial I\left(a_{1}\right)\right)>\beta(1 / 4-\beta / 2) / 5$.

For $x \in S$, we now define $f(x)=\lambda(x) e_{1}$.

(ii) Extend the definition of $f$ to points $x$ in the closure of $I\left(a_{1}, \ldots, a_{k}\right) \backslash$ $\bigcup I\left(a_{1}, \ldots, a_{k+1}\right)$ as follows: If $x_{0}$ is the center of the cube $I\left(a_{1}, \ldots, a_{k}\right)$, then $\beta^{-k}\left(x-x_{0}\right) \in S$, and we define:

$$
f(x)=f\left(\partial I\left(a_{1}, \ldots, a_{k}\right)\right)+2^{-(n[([k-1) / m]+1)+1)} \lambda\left(\beta^{-k}\left(x-x_{0}\right)\right) e_{((k-1) \bmod m)+1}
$$

(where $[(k-1) / m]$ is the greatest integer $\leq(k-1) / m)$.

4. Differentiability of $f:$ If $x, y \in I\left(a_{1}\right) \cap C(\beta)$, there exists a largest integer $K \geq 1$ such that $x, y \in I\left(a_{1}, \ldots, a_{K}\right)$, and so $|x-y| \geq \beta^{K}(1 / 4-\beta / 2)$. Thus

$$
|h(x)-h(y)| \leq M_{1} 2^{-(n / m) K}=M_{1} \beta^{K s} \leq M_{2}|x-y|^{s},
$$

which implies that $h$ is continuous; recalling the labelling requirements of parts 1 and 2 above, we see that $f$ is a continuous extension of $h$. Since $\left\|D^{p} f\right\|=0$ on the boundaries $\partial I\left(a_{1}, \ldots, a_{k}\right)$ for all $k, p \in \mathbf{Z}^{+}$, it follows furthermore that $f$ is $C^{\infty}$ on $I_{0} \backslash C(\beta)$. Now if $x \in I\left(a_{1}\right) \cap C(\beta), y \in I\left(a_{1}\right) \backslash C(\beta)$, and $K \geq 1$ is again the largest integer such that $x, y \in I\left(a_{1}, \ldots, a_{K}\right)$, it follows from the definition of $f$ that

$$
|f(x)-f(y)| \leq M_{1} 2^{-(n / m) K}=M_{1} \beta^{K s} \leq M_{2}|x-y|^{s} .
$$

This implies $\left\|D^{p} f\right\|=0$ on $C(\beta)$ for positive integers $p \leq P$. A direct calculation shows that for $y \in I\left(a_{1}, \ldots, a_{k}\right),\left\|D^{p} f(y)\right\| \leq M_{3}\left(2^{-(n / m) k} \beta^{-p k}\right)$, and so $\left\|D^{p} f\right\|$ tends to zero on approach to $C(\beta)$ provided $p \leq P$, which verifies that $f$ is $C^{P}$ on $I_{0}$. 
To see that $f$ is $C^{s}$, observe that since $f$ is $C^{\infty}$ on $I_{0} \backslash C(\beta)$, it suffices to show there exists a constant $M_{4} \geq 0$ such that

$$
\left\|D^{P} f(y)-D^{P} f(x)\right\| \leq M_{4}|y-x|^{s-P}
$$

for all $x \in C(\beta), y \in \bigcup I\left(a_{1}\right)$.

For $y \in C(\beta), D^{P}(y)=D^{P}(x)=0$, and so the inequality holds trivially. If $y \in \bigcup I\left(a_{1}\right) \backslash C(\beta)$, we see that for the smallest integer $j \geq 1$ such that $y \notin \bigcup I\left(a_{1}, \ldots, a_{j+1}\right)$,

$$
\begin{aligned}
\left\|D^{P} f(y)-D^{P} f(x)\right\| /|y-x|^{s-P} & =\left\|D^{P} f(y)\right\| /|y-x|^{s-P} \\
& \leq M_{4}\left(2^{-(n / m) j} \beta^{-j P} \beta^{-j(s-P)}\right) \\
& =M_{4}\left(2^{-(n / m)} \beta^{-s}\right)^{j}=M_{4},
\end{aligned}
$$

where $M_{4}=2^{n} M_{3}(1 / 4-\beta / 2)^{P-s}$. Thus $f$ is $C^{s}$.

Finally, because $f$ is locally of the form $f(x)=c+g(x) e_{((k-1) \bmod m)+1}$, where $c \in R^{m}$ and $g$ is a real-valued function, it clearly follows that rank $D f \leq 1$ on $I_{0}$. This concludes our construction.

\section{APPLiCATIONS}

With Theorem 1 and the preceeding construction at our disposal, we can now answer the following questions:

1. [Hirsch, 1976]: Let $U \subset \mathbf{R}^{3}$ and $V \subset \mathbf{R}^{2}$ be open sets. If $f: U \rightarrow V$ is $C^{1}$ and surjective, does $f$ necessarily have rank 2 at some point of $U$ ?

Answer: First note that if $f$ is also $C^{2}$, the question is answered affirmitively by the Morse-Sard Theorem. By Theorem 1, the answer is also "yes" for $C^{s+}$ maps with $s \geq 3 / 2$, but "no" for $C^{s}$ maps with $s<3 / 2$. In general, the image of a $C^{s+}$ rank 1 map $f: \mathbf{R}^{n} \rightarrow \mathbf{R}^{m}$ cannot have positive measure if $s \geq n / m$, whereas $C^{s}$ counterexamples exist for $s<n / m$.

2. We consider the following "dual" to Hirsch's question:

Let $U \subset \mathbf{R}^{4}$ and $V \subset \mathbf{R}^{3}$ be open sets. If $f: U \rightarrow V$ is $C^{1}$, singular and surjective, does $f$ necessarily have rank 1 at some point of $U$ ?

Answer: Although there is no known example of a map without rank 1 points which satisfies the conditions of the question, Corollary 2 asserts that such a map could not belong to the smoothness class $C^{4 / 3+}$.

3. [Norton, 1987]: For $s>1$ not an integer and $s(m-r)+r \leq n$ $<([s]+1)(m-r)+r$, does there exist a function $f \in C^{s}\left(\mathbf{R}^{n}, \mathbf{R}^{m}\right)$ and a $(s(m-r)+r)$-finite subset $B \subset \mathbf{R}^{n}$ of rank $r$ for $f$ such that $l_{m} f(B)>0$ ?

Answer: "Yes," provided $s<n / m$ (for $s=n / m$, the problem is still unresolved). As Norton notes, it suffices to verify the case $r=0$. Choose $s \in([n / m], n / m)$ and let $f$ be the $C^{s}$ function constructed in the previous section for $\beta=2^{-n / s m}$. A standard estimation technique (see [Falconer, 1985]) shows that the Hausdorff dimension of the Cantor set $C(\beta)$ is $-n \ln 2 / \ln \beta=s m$; to see that $\mathscr{H}^{s m} C(\beta)<\infty$, we observe that since $C(\beta)$ may be covered by $2^{n k}$ cubes of diameter $\alpha(k)=\beta^{k} \sqrt{n}$, we have:

$$
\mathscr{H}_{\alpha(k)}^{s m} C(\beta) \leq 2^{n k}(\alpha(k))^{s m}=n^{s m / 2}\left(2^{n} \beta^{s m}\right)^{k}=n^{s m / 2} .
$$

Letting $k \rightarrow \infty$ shows $\mathscr{H}^{s m} C(\beta) \leq n^{s m / 2}<\infty$. Finally, we recall that $f$ has 
rank 0 on $C(\beta)$ and maps $C(\beta)$ onto a set with nonempty interior, as desired.

\section{SOME QUESTIONS}

The inability of Theorem 1 to specify the behavior of singular $C^{s}$ maps for which $s=s_{0}$ suggests the following problem:

Q1. Given integers $n>m>r_{l} \geq 0$, let $s=\left(n-r_{l}\right) /\left(m-r_{l}\right)$. Does there exist a singular $C^{s}$ map $f: \mathbf{R}^{n} \rightarrow \mathbf{R}^{m}$ with subrank $r_{l}$ whose image contains an open set or at least has positive Lebesgue m-measure?

At present, the answer is known to be "no" only for the case $r_{l}=0, n / m \in$ $\mathbf{Z}^{+}$(see [Sternberg, 1964]).

A broader class of questions concerns establishing necessary rank conditions for a singular map with nonnull image in addition to those given in Corollary 2 . As an example, we pose the following generalization of the problem discussed in $§ I V .2$ as an open problem:

Q2. Given integers $n>m>r_{\max }>r_{l} \geq 0$, does there exist a $C^{1}$ map $f: \mathbf{R}^{n} \rightarrow \mathbf{R}^{m}$ such that $l_{m}\left(f\left(\mathbf{R}^{n}\right)\right)>0$ and $\operatorname{rank} D f(x) \in\left\{r_{\max }, r_{l}\right\}$ for all $x \in \mathbf{R}^{n}$, and if so, is the bound given in Theorem 1 realized by such maps?

Proving the existence of such maps reduces easily to the case $r_{l}=0$; note that our construction verifies the case $r_{\max }=1$.

\section{ACKNOWLEDGMENTS}

I am happy to thank Professors M. W. Hirsch and A. Norton for helpful criticisms of the manuscript.

Added in proof. In a recent article (Surjective mappings whose differential is nowhere surjective, Proc. Amer. Math. Soc. 111 (1991), 267-270), Y. Yomdin gives an interesting alternative construction of pathological subrank 1 singular mappings using the Whitney extension theorem. We note that Yomdin's open questions (1) and (3) are answered by our Theroem 1.

In an upcoming paper [Bates], we settle $Q 1$ of $\S \mathrm{V}$ above for all cases in the negative. Consequent improvements and extensions of Theorem 1 , as well as generalizations of these topics to infinite dimensions, will be described in the sequel to this note.

\section{REFERENCES}

[Bates] Towards a precise smoothness hypothesis in Sard's thoerem, Proc. Amer. Math. Soc. (to appear).

[Falconer, 1985] The geometry of fractal sets, Cambridge Tracts in Math. 85, Cambridge Univ. Press, Cambridge and New York.

[Federer, 1969] Geometric measure theory, Grundlehren Math. Wiss., vol. 153, Springer-Verlag, New York and Berlin.

[Hirsch, 1976] Differential topology, Graduate Texts in Math. vol. 33, Springer, New York and Berlin.

[Kaufman, 1979] A singular map of a cube onto a square, J. Differential Geom. 14, 593-594.

[Norton, 1986] $A$ critical set with nonnull image has large Hausdorff dimension, Trans. Amer. Math. Soc. 296, 367-376. 
[Norton, 1987] The fractal geometry of critical sets with nonnull image and the differentiablity of functions, Ph.d. Thesis, University of California, Berkeley.

[Sternberg, 1964] Lectures on differential geometry, Prentice-Hall, Englewood Cliffs, NJ.

[Whitney, 1935] A function not constant on a connected set of critical points, Duke Math J. 1, 514-517.

Department of Mathematics, University of California, Berkeley, California 94720 E-mail address: smbates@math.berkeley.edu 